Provided for non-commercial research and education use. Not for reproduction, distribution or commercial use.

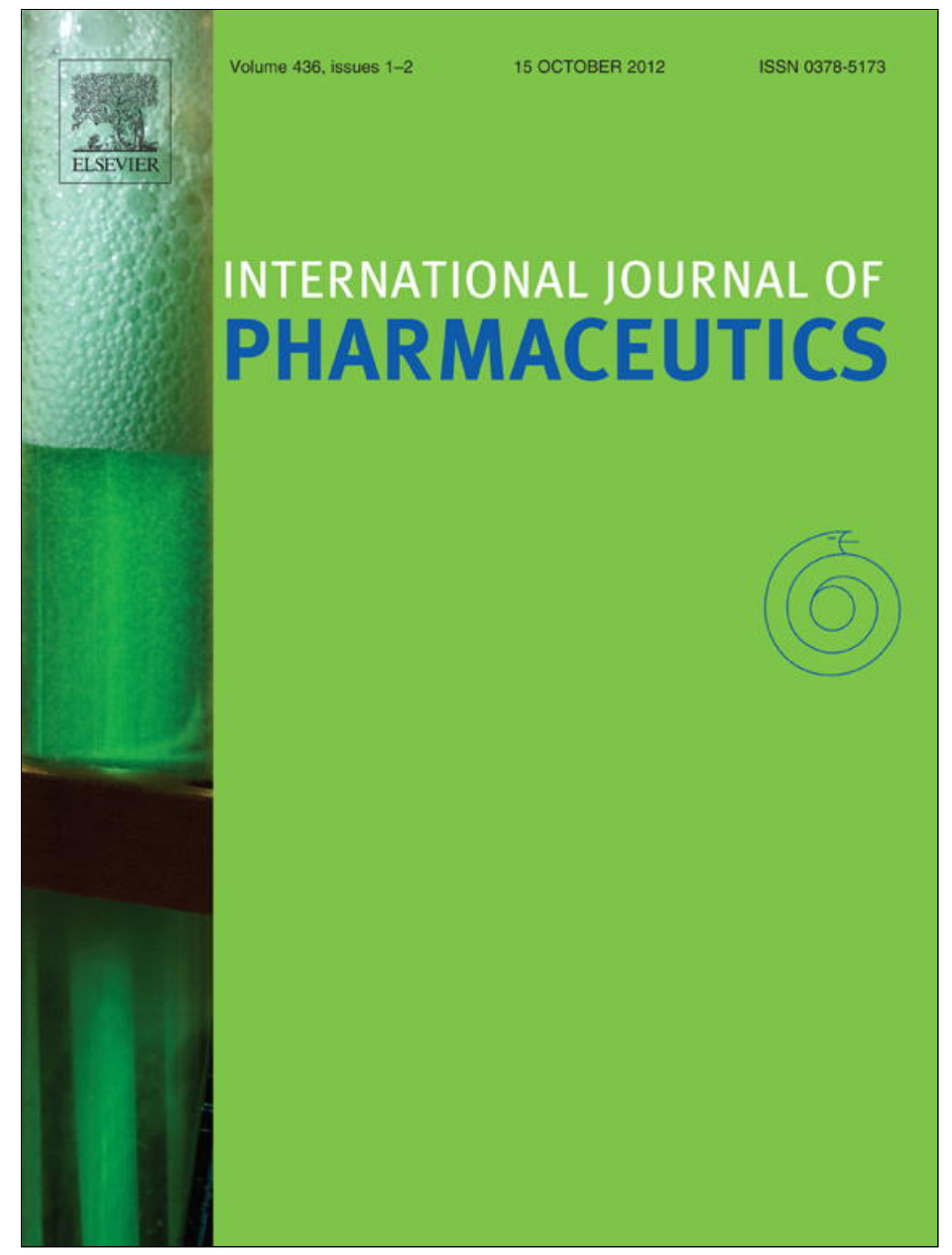

This article appeared in a journal published by Elsevier. The attached copy is furnished to the author for internal non-commercial research and education use, including for instruction at the authors institution and sharing with colleagues.

Other uses, including reproduction and distribution, or selling or licensing copies, or posting to personal, institutional or third party websites are prohibited.

In most cases authors are permitted to post their version of the article (e.g. in Word or Tex form) to their personal website or institutional repository. Authors requiring further information regarding Elsevier's archiving and manuscript policies are encouraged to visit:

http://www.elsevier.com/copyright 


\title{
Physicochemical characterization of GBV-C E1 peptides as potential inhibitors of HIV-1 fusion peptide: Interaction with model membranes
}

\author{
Maria Jesús Sánchez-Martín ${ }^{\mathrm{a}, *}$, Antonio Cruz ${ }^{\mathrm{b}}$, M. Antònia Busquets ${ }^{\mathrm{a}}$, Isabel Haro ${ }^{\mathrm{c}}$, \\ M. Asunción Alsina ${ }^{\mathrm{a}}$, Montserrat Pujol ${ }^{\mathrm{a}}$ \\ a Physical Chemistry Department, Faculty of Pharmacy, University of Barcelona, CSIC-Associated Unit: Peptides and Proteins: Physicochemical Studies, IN2UB Av. Joan XXIII s/n, 08028 \\ Barcelona, Spain \\ b Dept. de Bioquímica y Biología Molecular I, Facultad de Biología, Universidad Complutense, 28040 Madrid, Spain \\ ' Unit of Synthesis and Biomedical Application of Peptides, Department of Biomedical Chemistry, IQAC-CSIC, Jordi Girona 18, 08034, Barcelona, Spain
}

\section{A R T I C L E I N F O}

\section{Article history:}

Received 20 May 2012

Received in revised form 18 July 2012

Accepted 20 July 2012

Available online 31 July 2012

\section{Keywords:}

Hepatitis GB virus C

Synthetic peptide

Lipid monolayer

Compression isotherms

HIV-1 FP

Circular dichroism

\begin{abstract}
A B S T R A C T
Four peptide sequences corresponding to the E1 protein of GBV-C: NCCAPEDIGFCLEGGCLV (P7), APEDIGFCLEGGCLVALG (P8), FCLEGGCLVALGCTICTD (P10) and QAGLAVRPGKSAAQLVGE (P18) were studied as they were capable of interfering with the HIV-1 fusion peptide (HIV-1 FP). In this work, the surface properties of the E1 peptide sequences are investigated and their physicochemical characterization is done by studying their interaction with model membranes; moreover, their mixtures with HIV-1 FP were also studied in order to observe whether they are capable to modify the HIV-1 FP interaction with model membranes as liposomes or monolayers. Physicochemical properties of peptides ( $\mathrm{pI}$ and net charge) were predicted showing similarities between P7 and P8, and P10 and HIV-1 FP, whereas P18 appears to be very different from the rest. Circular dichroism experiments were carried out showing an increase of the percentage of $\alpha$-helix of P7 and P8 when mixed with HIV-1 FP corroborating a conformational change that could be the cause of their inhibition ability. Penetration experiments show that all the peptides can spontaneously insert into phospholipid membranes. Analysis of compression isotherms indicates that the peptides interact with phospholipids and the E1 peptides modify the compression isotherms of HIV$1 \mathrm{FP}$, but there is one of the peptides that excelled as the best candidate for inhibiting the activity of HIV-1 $\mathrm{FP}, \mathrm{P7}$, and therefore, that could be potentially used in future anti-HIV-1 research.
\end{abstract}

(c) 2012 Elsevier B.V. All rights reserved.

\section{Introduction}

The independently discovered human viruses GB virus C (GBVC) (Simons et al., 1995) and hepatitis G virus (HGV) (Linnen et al., 1996) are two isolates of the same single-stranded RNA virus. GBV$\mathrm{C}$ shows characteristics of a flavivirus-like genome, closely related to the hepatitis $C$ virus (HCV). The virus infects lymphocytes, but not hepatocytes and there is no conclusive evidence of a causal link between GBV-C and either acute or chronic liver disease. Recently, GBV-C has been investigated in the context of human immunodeficiency virus (HIV) infection and there are some reports finding that co-infection prolonged survival of patients and served as a potentially effective treatment (Tillmann and Manns, 2001; Xiang et al., 2001, 2004). Based on a co-infection model, GBV-C may influence HIV disease via inhibition of HIV by inducing chemokines, downregulating the HIV co-receptor(s), influencing cytokine profiles, or

\footnotetext{
* Corresponding author. Tel.: +34 9340245 58; fax: +34 934035987 E-mail addresses: mjesus_sanchez@hotmail.com, mjsanchez@ub.edu (M.J. Sánchez-Martín).
}

having other - as yet undefined - effects on the host lymphocytes (Xiang et al., 2001, 2004). However, the mechanism responsible for the beneficial effect that the GBV-C virus has on the course of disease caused by HIV has not yet been identified (Bhattarai and Stapleton).

We are currently examining the capacity of GBV-C synthetic peptides to interact and to induce fusion in model membranes (Sánchez-Martín et al., 2009, 2010) The capacity, observed in vitro, to inhibit the leakage of vesicular contents caused by the HIV-1 fusion peptide (HIV-1 FP) is the property that allows us to select the sequences of the envelop protein $\mathrm{E} 1$ of GBV-C/HGV in order to study their interaction with model membranes and with HIV-1 FP.

In this work, four peptides corresponding to the E1 protein of GBV-C are selected as possible inhibitors of the activity of HIV-1 FP (Sánchez-Martín et al., 2011a).

The aim of this work is to study the effect of the E1 peptides on the activity of the peptide sequence that represents the 23mer N-terminal domain of the surface protein gp41 of HIV which corresponds to HIV-1 FP.

Surface properties are among the most important features of biomaterials. Surface functional groups and their arrangement in 
the nanoscale topology of the material surface have dramatic effects on amount, orientation, and conformation of adsorbed proteins. For these reasons, adsorption and conformational change of proteins on biomaterial surfaces are widely considered to be one of the most important mechanisms controlling interactions between biomaterials and the surrounding biological system.

Small variations in primary structure strongly affect the local predisposition toward secondary structure in smaller peptides. $\alpha$ Helix (Parthasarathy et al., 1995), $\beta$-sheet (Ramirez-Alvarado et al., 1997), and a variety of turns (Perczel and Hollosi, 1996) have been reported for short peptides. These are consistently found to depend strongly on the primary structure. For example, in model $\alpha$-helical peptides, favorable intramolecular electrostatic interactions arising from charged side chains are frequently used to stabilize $\alpha$-helicity (Andrews and Tabor, 1999). In contrast, polypeptides lacking such specific interactions follow the entropic bias toward a "random coil" conformation, in which the average dihedral angles follow local torsional energetic, independent of surrounding residues (Brant and Flory, 1965).

As a continuation of a previous works carried out in our group (Sánchez-Martín et al., 2011a), the surface properties of the E1 peptide sequences are investigated and their physicochemical characterization is done by studying their interaction with model membranes; lipopeptide interactions with lipid monolayers of 1,2-dipalmitoyl-sn-glycero-3-phosphocholine (DPPC), 1,2dimyiristoyl-sn-glycero-3-[phospho-rac-(1-glycerol)] (DMPG) and 1,2-dipalmitoyl-sn-glycero-3-phospho-rac-(1-glycerol) (DPPG) are studied.

In addition, conformational analysis by circular dichroism was carried out in presence of liposomes as model membranes. Moreover, we are interested in study the conformational changes when HIV-1 FP is in presence of E1 peptides in order to know if it is one of the reason for inhibiting its activity.

\section{Experimental}

\subsection{Materials}

1,2-Dimyiristoyl-sn-glycero-3-[phospho-rac-(1-glycerol)] (DMPG) and 1,2-dipalmitoyl-sn-glycero-3-phospho-rac-(1glycerol) (DPPG) and 1-palmitoyl-2-oleoyl-sn-glycero-3phosphocholine (POPG) were purchased from Avanti Polar Lipids. Their purity was higher than $99 \%$ and they were used without further purification.

Chloroform and methanol were purchased from Merck. Water was double distilled and deionised (MilliQ system, Millipore) (18.2 $\mathrm{M} \Omega \mathrm{cm}, \mathrm{pH} 5.8$ ). Buffer in all experiments was HEPES (from Sigma-Aldrich) $5 \mathrm{mM}$ and $\mathrm{NaCl}$ (from Merck) $20 \mathrm{mM}$, pH 7.4.

\subsection{Methods}

\subsubsection{Peptides synthesis}

The peptides corresponding to E1 protein of GBV-C: NCCAPEDIGFCLEGGCLV (P7), APEDIGFCLEGGCLVALG (P8), FCLEGGCLVALGCTICTD (P10) and QAGLAVRPGKSAAQLVGE (P18), and the HIV-1 FP, AVGIGALFLGFLGAAGSTMGAAS, were obtained by solid-phase methodologies and purified by preparative high performance liquid chromatography, as previously described (Sánchez-Martín et al., 2011a).

\subsubsection{Preparation of multilamellar vesicles}

1-Palmitoyl-2-Oleoyl-sn-Glycero-3-Phosphocholine (POPG) and peptides were dissolved in a chloroform:methanol $(2: 1,(\mathrm{v} / \mathrm{v}))$ mixture. Different mixtures of POPG and E1 peptides with or without HIV-1 FP were done and the solutions were evaporated to dryness in vacuum with a rotary evaporator. The dried lipid film was subjected to a high vacuum overnight to remove trace amounts of solvent. Then, the lipid films were hydrated with double distilled and deionised water to obtain multilamellar vesicles (MLVs).

\subsubsection{Bioinformatic analysis tools for peptide characterization}

Physicochemical properties of peptides ( $\mathrm{p} I$ and net charge) were predicted using the Isoelectric Plot calculation program at http://www.bioinformatics.org/JaMBW//3/1/6/index.html. Hydrophobicity profiles were obtained by means of a calculation program at http://www.vivo.colostate.edu/molkit/hydropathy/index.html, using the Hopps and Woods scale with a window size of 6 .

\subsubsection{Circular dichroism}

CD spectra were recorded on a Jasco J-810 spectropolarimeter (Japan Spectroscopic Company, Tokyo). All measurements were done in water. Cells $1 \mathrm{~cm}$ in diameter were used and the spectra were measured between 170 and $250 \mathrm{~nm}$ using a spectral bandwidth of $1 \mathrm{~nm}$ and a scan speed of $10 \mathrm{~nm} / \mathrm{min}$.

All measurements were performed at $25^{\circ} \mathrm{C}$ and the data were expressed in terms of mean residue ellipticities $[\theta]\left(\operatorname{deg} \mathrm{cm}^{2} \mathrm{dmol}^{-1}\right)$. Three scans were accumulated to improve the signal to noise ratio. Before reading the peptide spectra, a spectrum of the blank solution was subtracted and the data converted to mean residue ellipticity units. (Greenfield, 2006) Moreover, Contin by the Dichroweb server at http://dichroweb.cryst.bbk.ac.uk/html/home.shtml program was used to treat experimental CD results (Lobley et al., 2002; Whitmore and Wallace, 2004).

The percentage of $\alpha$-helix conformation in the peptides was estimated using the formalism of Chen et al. (1974) This approach assumes that the maximum theoretical ellipticity for a given peptide or protein at $222 \mathrm{~nm}$ may be derived from the number of amino acid residues $n$, and the ellipticity at $222 \mathrm{~nm}$ of a helix of infinite length described by Eq. (1).

$$
\% \alpha-\text { helix }=\frac{[\theta]_{222}}{[-39500(1-2.75 / n)]}
$$

\subsubsection{Adsorption at the air-water interface}

Adsorption studies at the air-water interface were carried out using a NIMA Langmuir Film Balance equipped with a Wilhelmy platinum plate (Nima Technology, Coventry) and a Teflon trough that was rinsed with ethanol and distilled water before use. All experiments were performed at room temperature.

The surface activity of peptides was first studied to determine the equilibrium spreading pressure. Using a cylindrical PTFE trough $\left(19.6 \mathrm{~cm}^{2}, 27.2 \mathrm{~cm}^{3}\right)$, increasing volumes of a $0.28 \mathrm{mM}$ peptide solution were injected below HEPES subphase ( $\mathrm{pH} 7.4)$ through a lateral hole and the adsorption of the peptide at the air/water interface was therefore monitored by following the increase in surface pressure as a function of time under continuous stirring of the subphase.

\subsubsection{Insertion of peptides into monolayers}

The kinetics of insertion of the peptides into monolayers of DMPG and DPPG were measured using the same trough as for the surface activity. For these experiments, a lipid stock solution was prepared and added drop wise on the subphase until the desired lipid pressure was achieved. After 10-20 min the equilibrium of the lipid monolayer was reached. Then, a $0.28 \mathrm{mM}$ peptide solution was injected into the subphase through the side hole of the trough. The subphase was magnetic stirred during the measurements and surface pressure changes were monitored as function of time until it remained constant. 


\subsection{Compression isotherms}

Compression isotherms were carried out on a Nima (UK) Langmuir Teflon trough (surface area $595 \mathrm{~cm}^{2}$, volume $280 \mathrm{~cm}^{3}$ ). By depositing appropriate volumes of chloroform stock solutions of phospholipids $(0.28 \mathrm{mM})$ and of peptides $(0.28 \mathrm{mM})$, the lipid-peptide spreading solutions were obtained. Monolayers were formed by applying small drops of the spreading solutions on the HEPES subphase ( $\mathrm{pH}$ 7.4) with a micro syringe (Hamilton Co., Reno, $\mathrm{NV}$ ). After $15 \mathrm{~min}$, monolayers of the desired composition were continuously compressed with an area reduction rate of $15 \mathrm{~cm}^{2} \mathrm{~min}^{-1}$. The films were compressed to their collapse pressure when possible. Each run was repeated three times and the reproducibility was $\pm 1 \AA^{2}$ molecule $^{-1}$.

\subsubsection{Epifluorescence experiments}

Surface pressure-area measurements and microscopic observations of phospholipid monolayers were performed using a specially designed Langmuir-Blodgett (LB) trough $\left(200 \mathrm{~cm}^{2}\right.$, Nima Technology, Inc., Coventry, UK) equipped with a continuous Teflon-ribbon barrier able to sustain maximal surface pressures with no leakage, and thermostated at the desired temperature. The trough was set inside a custom-made closed chamber to ensure isolation from external light. Films were transferred onto a Menzel-Gläser $24 \mathrm{~mm} \times 60 \mathrm{~mm}$ cover slips at a rate of substrate movement of $5 \mathrm{~mm} / \mathrm{min}$ while the film was compressed to obtain the complete $\pi-A$ isotherm as described previously (Wang et al., 2007). The entire transferred film typically occupied a length of $\sim 20 \mathrm{~mm}$ on the substrate. $\pi-A$ isotherms were simultaneously recorded during the transfer.

To form monolayers, the lipids DPPC and DPPG, and their mixtures with peptides ( $5 \%$ with respect to phospholipid, $\mathrm{mol} / \mathrm{mol}$ ) were prepared in chloroform/methanol $(3: 1,(\mathrm{v} / \mathrm{v}))$, and $1 \mathrm{~mol} \%$ of NBD-PC was included. Monolayers were spread by depositing very small aliquots of the chloroform/methanol solutions on a double distilled water subphase. After spreading of a monolayer, the organic solvent was allowed to evaporate for $5 \mathrm{~min}$, and the monolayer was compressed at $25 \mathrm{~cm}^{2} \mathrm{~min}^{-1}$ to a surface pressure of $3 \mathrm{mN} / \mathrm{m}$ and let to stabilize for $10 \mathrm{~min}$. Then, the compression isotherm was registered.

The transferred monolayers were observed in a Leica DM4000B microscope (Leica Microsystems, Wetzlar, Germany) equipped with the appropriate fluorescence filters to allow for the observation of NBD-PC fluorescence (maximum fluorescence excitation at $480 \mathrm{~nm}$ and emission at $527 \mathrm{~nm}$ ). Images were obtained at different positions from the transferred films and subsequently assigned to the surface pressures obtained during film transfer (Wang et al., 2007).

\section{Results and discussion}

\subsection{Bioinformatic analysis tools for peptide characterization}

Physical properties such as the isoelectric point $(\mathrm{p} I)$ or the net charge at $\mathrm{pH} 7.4$ ( $\mathrm{pH}$ of the study) of the peptides were predicted in order to know whether the behavior of the different sequences is related to these properties. Results are shown in Table 1.

P7 and P8 present similar values of both properties, whereas the $\mathrm{pI}$ and the charge of P10 are the most close to those of HIV-1 FP. In the contrary, P18 differs much from the rest.

\subsection{Circular dichroism}

In order to gain insights into the differences between peptides and their interaction with HIV-1 FP, a conformational study was carried out. Circular dichroism experiments and the corresponding
Table 1

Prediction of the Isoelectric point and the net charge at pH 7.4 for HIV-1 FP and E1 synthetic peptides.

\begin{tabular}{lll}
\hline & Isoelectric point & Net charge at $\mathrm{pH} 7.4$ \\
\hline HIV-1 FP & 6 & -0.1 \\
P7 & 2.88 & -3.5 \\
P8 & 2.88 & -3.2 \\
P10 & 3.18 & -1.5 \\
P18 & 11.38 & +2 \\
\hline
\end{tabular}

quantitative analysis of the experimental data using a deconvolution computer program were done. In general, short synthetic peptides do not have a preferential conformation in solution, but can sometimes adopt moderately stabilized secondary structures (Pérez et al., 1998). CD experiments were carried out in presence of POPG vesicles as it was found that HIV-1 bound to negative phospholipids stronger than to lipids devoid of a net charge (Wu et al., 2002). Firstly, CD spectra of the peptides separately were recorded. Afterwards, E1 peptides were incubated with HIV-1 FP and added to the liposomes solution.

In this work, we used CD to analyze if the ability of the E1 peptides to bind HIV-1 FP induces a conformational change in its secondary structure. In this sense, CD spectra of the HIV-1 FP in presence of POPG liposomes showed a negative band with a peak around $215 \mathrm{~nm}$ which could be attributed to a $\beta$-structure contribution probably due to peptide aggregation (Fig. 1). In this medium, the peptide spectrum also showed a negative band near $195 \mathrm{~nm}$ that is typical of random coil conformation.

On the other hand, in P7, P8 and P18 spectra, the characteristic positive band around $195 \mathrm{~nm}$ appeared showing the spectra like those of an $\alpha$-helix structure, and they also show a negative band with a peak between 215 and $220 \mathrm{~nm}$ which could be attributed to a $\beta$-structure contribution. CD spectra of P10 also showed a negative band near $195 \mathrm{~nm}$, typical of random coil conformation.

The experimental $\mathrm{CD}$ spectrum of the mixture of $\mathrm{E} 1$ peptides and HIV-1 FP in aqueous solution is different from the theoretical spectrum obtained by summing experimental spectra of equivalent amounts of the peptides alone (dotted spectra in Fig. 1); thus, the results suggest that the E1 peptides interact with HIV-1 FP. The mixture demonstrated that the addition of E1 peptides to the HIV-1 FP decreased the mean residue ellipticity at $215 \mathrm{~nm}$, thus decreasing the percentage of $\beta$-structure. Therefore, E1 peptides seem to inhibit the aggregation propensity when mixed with HIV-1 FP, showing an interaction between the peptides.

Table 2 shows the estimation, from the CD spectra, of the content of $\alpha$-helical conformation of the peptides according to molar ellipticity at $222 \mathrm{~nm}$ (calculated from Eq. (3)). These results slightly differ from the obtained in a previous work (Sánchez-Martín et al., 2011b) where we performed a CD study of these peptides to know the capacity of them to adopt secondary structure in an hydrophobic environment. Now we use POPG vesicles instead of

Table 2

Estimation, from the CD spectra, of the content of $\alpha$-helical conformation of the HIV-1 FP and E1 peptides according to molar ellipticity at $222 \mathrm{~nm}$.

\begin{tabular}{lc}
\multicolumn{2}{l}{$\% \alpha$-helix in presence of POPG liposomes } \\
\hline & {$[\theta]_{222}$} \\
\hline HIV-1 FP & 5.2 \\
P7 & 7.7 \\
P7 + HIV-1 FP & 9.3 \\
P8 & 6.1 \\
P8 + HIV-1 FP & 6.4 \\
P10 & 5.2 \\
P10 + HIV-1 FP & 7.2 \\
P18 & 11.6 \\
P18 + HIV-1 FP & 6.4 \\
\hline
\end{tabular}



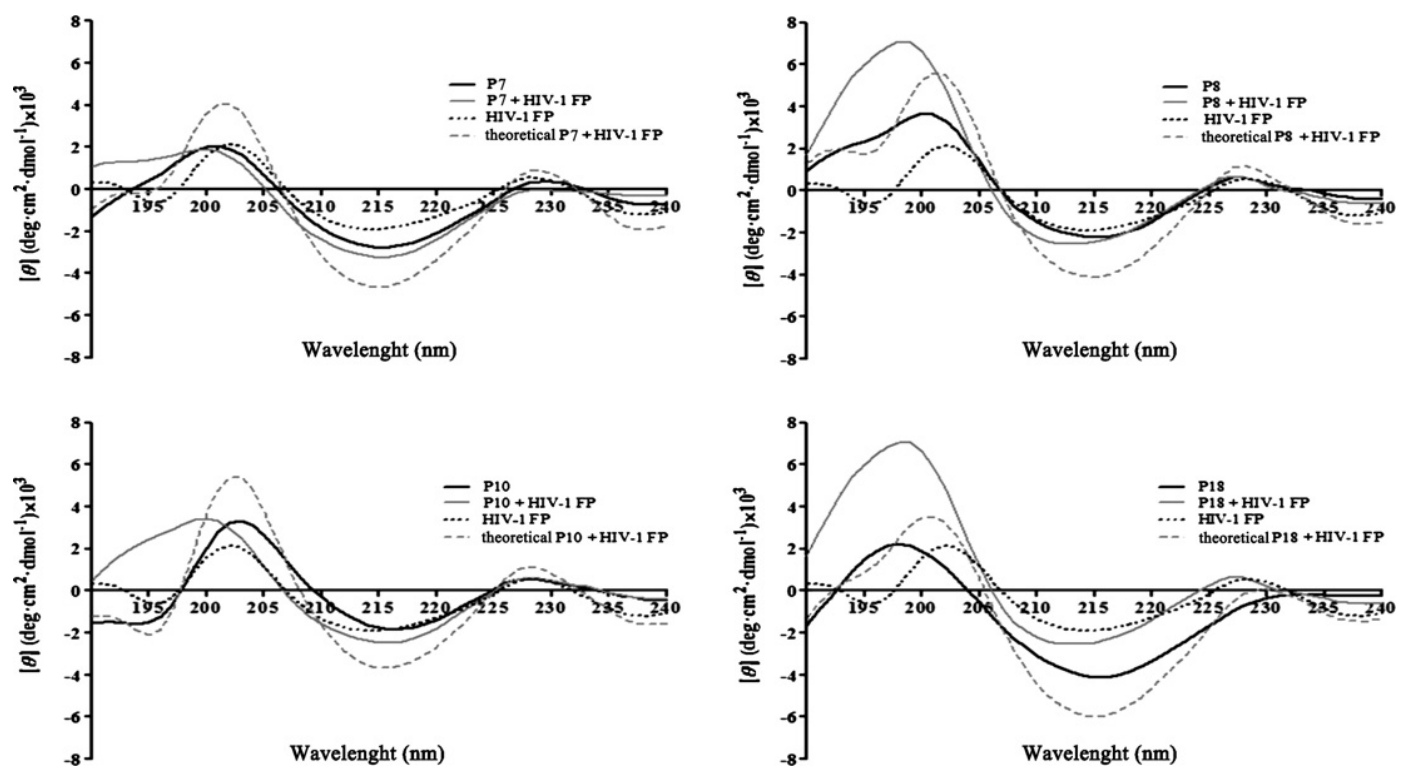

Fig. 1. CD spectra of E1 synthetic peptides and HIV-1 FP in presence of POPG liposomes in a Hepes buffer solution, recorded at $298 \mathrm{~K}$.

trifluoroethanol (TFE), an halogenated alcohol that it is known induces $\alpha$-helical conformation in peptides. So the $\alpha$-helical content is lower in this case. On the other hand this is a normal behavior observed in other E1 peptides that were studied (Fernández-Vidal et al., 2008).

First, results show that P18 has a high percentage of $\alpha$-helix compared to the rest and the percentage obtained for P10 is equal to that obtained for HIV-1 FP; these results are coherent with those obtained in Table 1, showing that P10 and P18 behave different from P7 and P8, and that P10 has some similarities with HIV-1 FP. This similarity obtained here corroborates the results obtained in a previous work where we observed that P10 could be an internal fusion peptide of GBV-C, as HIV-1 FP, and it was discarded as a possible inhibitor of HIV-1 FP. P18 is the peptide that presents the highest change in the conformation when mixed with the HIV-1 FP, showing a clear interaction between both peptides.

\subsection{Adsorption at the air-water interface}

Fig. 2 shows the adsorption isotherm profile for the E1 synthetic peptides. A small gradual adsorption of peptide was observed at low peptide concentration. The higher the peptide concentration in the subphase the faster the incorporation process and the higher the surface pressure attained. The shape of the surface activity curves

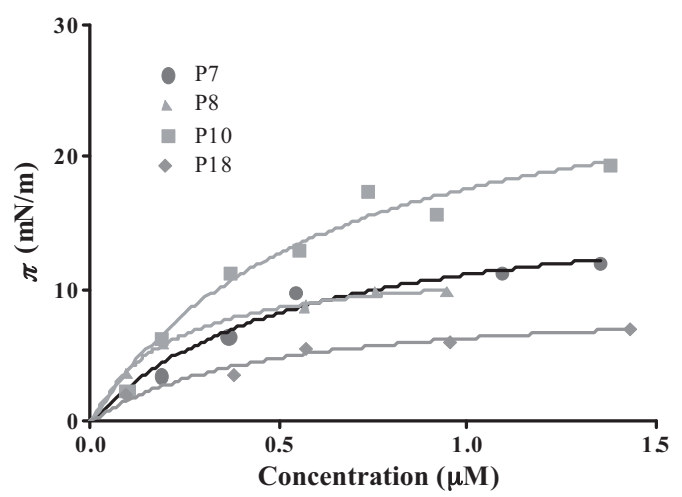

Fig. 2. Surface activity curve of E1 synthetic peptides. approximates a rectangular hyperbola and it was fitted to Eq. (2) via non-linear least squares regression analysis.

$\pi=\frac{C \pi_{\max }}{K+C}$

where $C$ is the concentration, $\pi_{\max }$ is the maximum pressure achieved and $K$ is a characteristic constant equal to the peptide concentration that yields $(1 / 2) \pi_{\max }$. Fitting the data, the values obtained are shown in Table 3. These $K$ values were chosen to further penetration studies, as $K$ corresponds to the suitable concentration of the peptide that should be used in the bulk subphase for experiments of penetration kinetics, lower than the equilibrium spreading pressure of the peptide (Rafalski et al., 1990).

By applying the Gibbs adsorption equation in its simpler form (Eq. (3)) it is possible to calculate the peptide surface excess concentration $(\Gamma)$

$\Gamma=\frac{\Delta \pi}{R T \Delta \ln c}$

where $R$ is the gas constant $\left(8.31 \mathrm{~J} \mathrm{~K}^{-1} \mathrm{~mol}\right), T$ is the temperature $(298 \mathrm{~K}), \Delta \pi$ is the maximum pressure increase achieved for each peptide concentration and $C$ is the peptide concentration.

The surface excess concentration of the E1 synthetic peptides at saturation $\left(\Gamma_{\max }\right)$ deduced from the slope of the $\Delta \pi-\ln C$ curve (Eq. (3)) allows us to calculate the surface molecular area by means of Eq. (4), where $N$ is Avogadro's constant. Results are shown in Table 3.

$A=\frac{1}{\Gamma_{\max } N}$

Clearly, the four peptides decrease the surface tension of the air-water interface. However, their hydrophobic profiles based on their primary sequence are very different and this fact induces different behaviors. The main difference is the greater decrease in tension induced by $\mathrm{P} 10$, this suggests that P10 develops stronger hydrophobic interactions than the others when engaged in an airwater interface most likely because they adopt different structural states. This fact is corroborated with the hydrophobicity plot of P10 obtained using Hopp-Woods scale that was designed for predicting potentially antigenic regions of polypeptides. In this type of plots, values greater than 0 are hydrophilic and thus likely to be exposed on the surface of a folded protein. For P10 all the values in the plot are negative, showing a high hydrophobicity profile, as HIV-1 FP 
Table 3

Analysis of the adsorption isotherms of the E1 synthetic peptides. $\pi_{\max }$ is the maximum pressure achieved and $K$ is a characteristic constant equal to the peptide concentration that yields $(1 / 2) \pi_{\max } \Gamma_{\max }$ is the surface excess concentration at saturation and $A$ is the molecular area.

\begin{tabular}{llllr}
\hline & $\pi_{\max }\left(\mathrm{mN} \mathrm{m}^{-1}\right)$ & $K(\mu \mathrm{M})$ & $r^{2}$ & $\Gamma_{\text {max }}\left(\times 10^{22}\right.$ res m $\left.^{-2}\right)$ \\
\hline P7 & 17.22 & 0.55 & 0.953 & 1.85 \\
P8 & 12.14 & 0.21 & 0.993 & 1.20 \\
P10 & 28.49 & 0.62 & 0.966 & 2.79 \\
P18 & 9.20 & 0.46 & 0.947 & 0.34 \\
\hline
\end{tabular}

also presenting negative values in the plot. P7 and P8 have negative values from the residues 8 to 15 and 4 to 15 , respectively, and P18 shows negative values for the residues 3 to 5 and 14 to 15 , being the less hydrophobic peptide and corroborating the low $\pi_{\max }$ value obtained at the adsorption isotherm.

\subsection{E1 synthetic peptides insertion into phospholipid monolayers}

The ability of the E1 peptides to insert into phospholipid monolayers spread at the air-water interface was monitored by measuring variations in surface tension using different initial pressures, and by injecting a given peptide concentration in the subphase. The selected concentration corresponds to the $K$ values calculated in Section 3.3. Various penetration experiments were carried out using two different phospholipids, DMPC and DMPG, where the nature of the headgroups (zwitterionic and negatively charged) is different. For both lipids, the general trend observed is that the greater $\pi_{i}$, the lower the degree of incorporation of the peptide into the monolayer because of the closer packing of the lipids at higher initial pressures (Fig. 3).

The monolayer exclusion pressure $\pi_{\mathrm{e}}$ (that is, the surface pressure above which the peptide does not penetrate into the monolayer) was obtained by extrapolating the plot to $\Delta \pi=0 \mathrm{mN} \mathrm{m}^{-1}$ (Bougis et al., 1981). It can be seen that the peptide interacts with the two lipids tested; the nature of the headgroups has influence on penetration for all peptides except for P18. DMPG form anionic monolayers while monolayers of DMPC are zwitterionic. The insertion of the peptides in the anionic monolayers is lower than in the zwitterionic, probably due to the negative charge of the peptides; however, P18, that is positively charged, interacts in the same way with both phospholipids. The $\pi_{\mathrm{e}}$ is similar for all the peptides except for P10 that has a $\pi_{\mathrm{e}}$ for DMPC higher than the others peptides, indicating a better uptake by this zwitterionic phospholipid.

\subsection{Spreading at the air-water interface: compression isotherms}

The compression isotherms for the E1 peptides and their mixtures with HIV-1 FP when mixed with DMPC and DMPG were obtained. We could observe how the shape of the isotherms of HIV-1 FP and phospholipids changes drastically when HIV-1 FP is mixed with any of the other E1 peptides (figure not shown). The isotherms were analyzed by examining the variation of the mean molecular area (that of the contributions of both the peptide and the lipid) as a function of the peptide/lipid ratio at a given and constant surface pressure. These variations are reported in Fig. 4 and correspond to a pressure of $10 \mathrm{mN} / \mathrm{m}$, although for 5,20 and $30 \mathrm{mN} / \mathrm{m}$ the same trends are observed for all the peptides. Analysis of the plots reveals several similar situations in both lipid-peptide combinations. First, they show a nonlinear behavior for all the peptides and their mixtures, indicating that all the peptides are miscible and interact with the phospholipids assayed (Gaines, 1966). The mixed monolayers present positive deviations of ideality, at any peptide molar fraction assayed, except when HIV-1 FP is mixed with P7, suggesting that the peptides interact with phospholipids through repulsive interactions (Galvez and Cabrerizo, 1991). Although all the E1 peptides decrease the deviations caused by HIV-1 FP, P7 is the only one that make these deviations become negative and, at lower $\mathrm{X}_{\mathrm{HIV}-1 \mathrm{FP}+\mathrm{P} 7}$, they show an ideal behavior. The contraction observed in the case of P7 indicates that peptide-lipid interactions are attractive and may reflect the formation of peptide-lipid complexes through hydrophobic interactions (Deshayes et al., 2004).
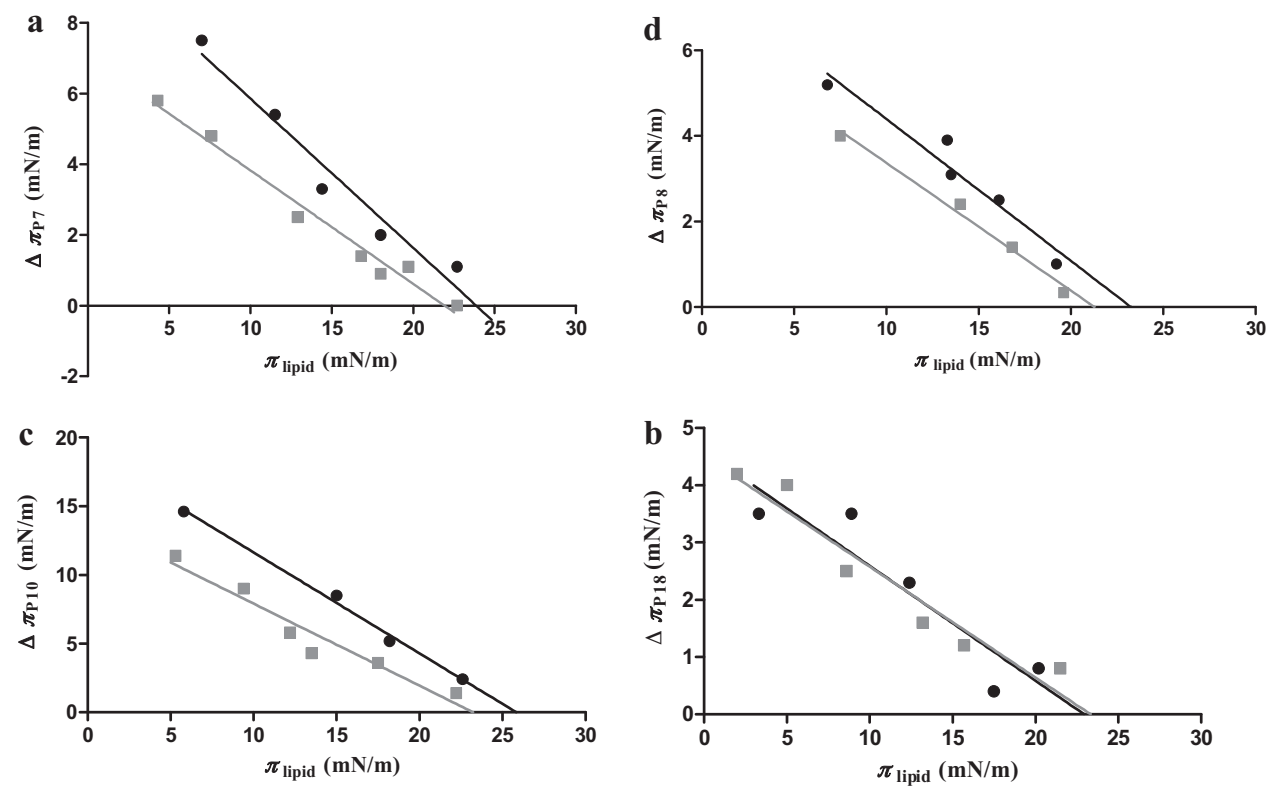

Fig. 3. Surface pressure increase $(\Delta \pi)$ caused by E1 synthetic peptides in monolayers of different phospholipids in front of the lipid initial pressure ( $\left.\pi_{i}\right)$. Grey squares correspond to DMPG and black circles to DMPC. (a) P7, (b) P8, (c) P10 and (d) P18. 

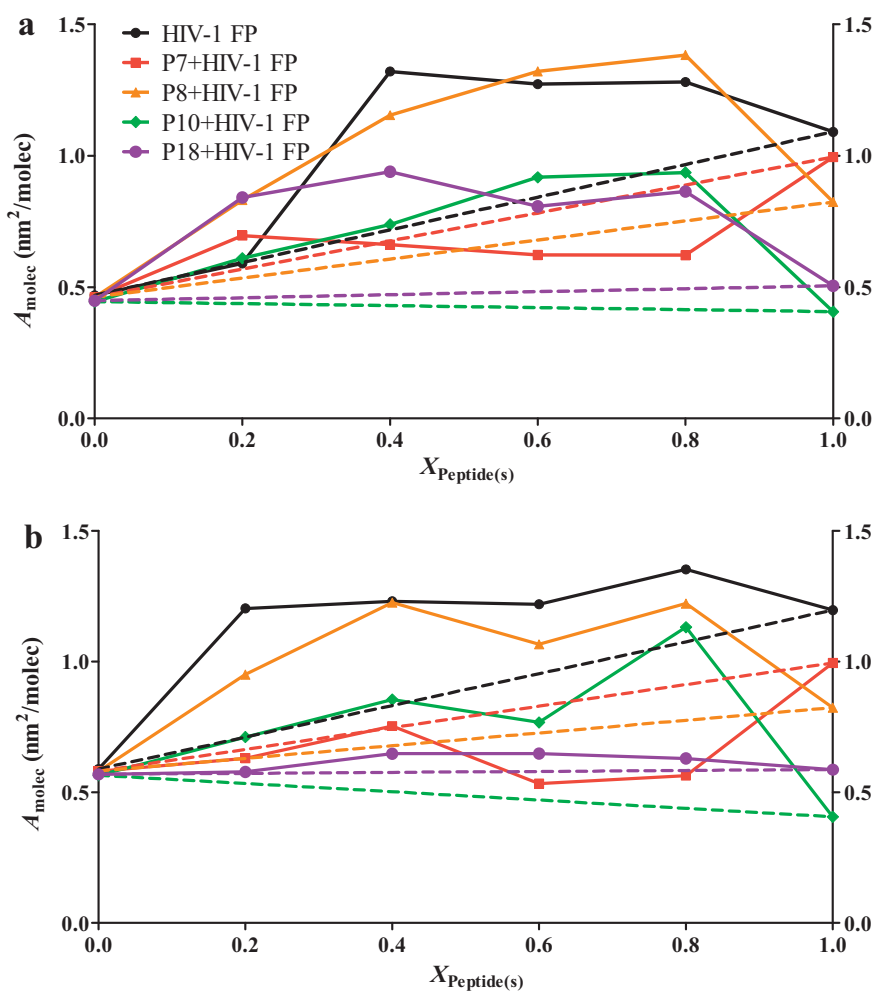

Fig. 4. Plots of $A_{\text {molec }}$ for the mixtures of E1 peptides and HIV-1 FP as a function of the peptides molar fractions for mixed monolayers of: (a) DMPC and (b) DMPG. Dotted lines indicate ideality.

The deviations from ideality of the mixed monolayers of P18 with DMPG are smaller than for the other peptides, this behavior is related with the positive charge of P18 and, therefore, the electrostatic attraction towards DMPG. These results are coherent with those observed in previous assays where P7 stood out as one with the highest capacity to inhibit the fusion peptide activity (SánchezMartín et al., 2011a).

\subsection{Compression isotherms: epifluorescence experiments}

To further characterize the lipid physical state of the monolayers in presence and in absence of the peptides, mixed monolayers of DPPC and DPPG with P7 and HIV-1 FP were compressed and transferred to a solid substrate in order to observe them by epifluorescence microscopy. Fig. 5 shows the surface pressure-area per molecule $(\pi-A)$ isotherms of DPPC (Fig. 5a) and DPPG (Fig. 5b) mixed with P7, HIV-1 FP and the mixture P7: HIV-1 FP (2:1). The isotherms showed that the presence of P7 or HIV-1 FP in the subphase caused an expansion of the DPPC film, suggesting that the peptide is occupying some space in the interface, or, at least it is interacting with the phospholipid monolayer sufficiently to perturb the usual lipid packing. The mixture of both peptides showed an smaller expansion than when peptides are alone, demonstrating an interaction between both peptides. In the case of DPPG, the presence of the peptides caused a compression of the DPPG film, but again we could observe that when mixing P7 and HIV-1 FP the effect of HIV-1 FP is drastically reduced.

Microscopic observations of DPPC monolayers containing $1 \mathrm{~mol} \%$ NBD-PC transferred to glass cover slips showed the typical domain coexistence along the phase transition beginning around $9 \mathrm{mN} / \mathrm{m}$ and observed as a plateau in the $\pi-A$ isotherm (Fig. 6). Liquid expanded and liquid condensed (LE-LC) coexistence regions are observed as dark LC domains excluding the fluorescent probe and bright green areas of LE phase similar to those previously observed in situ at the Langmuir through (Nag et al., 1991, 1996; Perez-Gil et al., 1992).

When $5 \%$ of $\mathrm{P} 7$ peptide is added to the monolayer composition, a matrix of a branched structure could be observed in the monolayer at low pressures, probably due to the presence of the peptide in the interphase. At higher pressures where the phase transition starts, this net is less evident indicating a partial remixing of the peptide in the lipid bulk. Indeed, the peptide produces a change in the lipid phase transition, reducing the size and increasing the number of condensed domains, with a less effect in the total condensed phase at any surface pressure. The presence of peptide could induce an increase in the nucleation points where the condensed domains grow. This effect can also explain the presence of small condensed domains at pressures below the one where the phase transition starts in monolayers made with lipid alone.

The addition of HIV-1 FP to DPPC monolayers had a similar effect producing a decrease in the domain size. In contrast to P7, HIV-1 FP does not seem to increase the number of condensed domains and slightly increases the surface pressure where the domains start no nucleate. At higher pressures the domains grow with shapes similar to the observed in the lipid monolayers but with less average condensed areas than the observed in the DPPC monolayer. The combination of both peptides produces an intermediate situation where effects of the presence of P7 and HIV-1 FP can be observed. P7 produce an initial peptide network an also reduce the size of the condensed domains but the effect of HIV-1 FP can also be observed as reduction of the number and of domains and total condensed area. At higher pressures (see Fig. 6 at $\sim 18 \mathrm{mN} / \mathrm{m}$ ), structures similar to the observed in presence of P7 or HIV-1 FP alone can be observed in the same epifluorescence frame, suggesting a separate effect of each peptide in DPPC monolayers.

The effect of the peptides in the structure of monolayers of DPPG was also analyzed by epifluorescence microscopy (Fig. 7). The monolayers of DPPG showed a phase transition from liquid
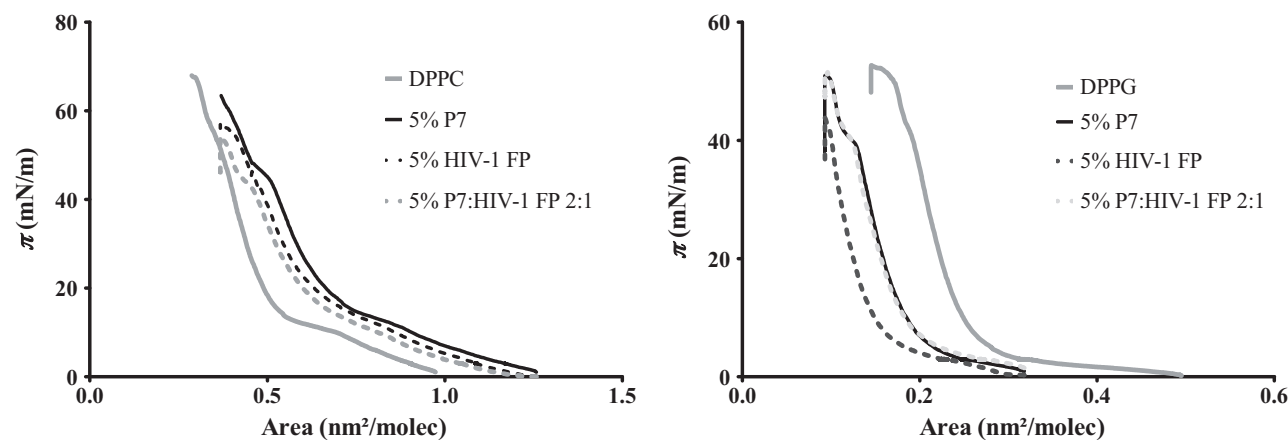

Fig. 5. Surface pressure-mean area per molecule $(\pi-A)$ compression isotherms of (a) DPPC and (b) DPPG monolayers mixed with P7, HIV-1 FP and their mixture 2:1. 


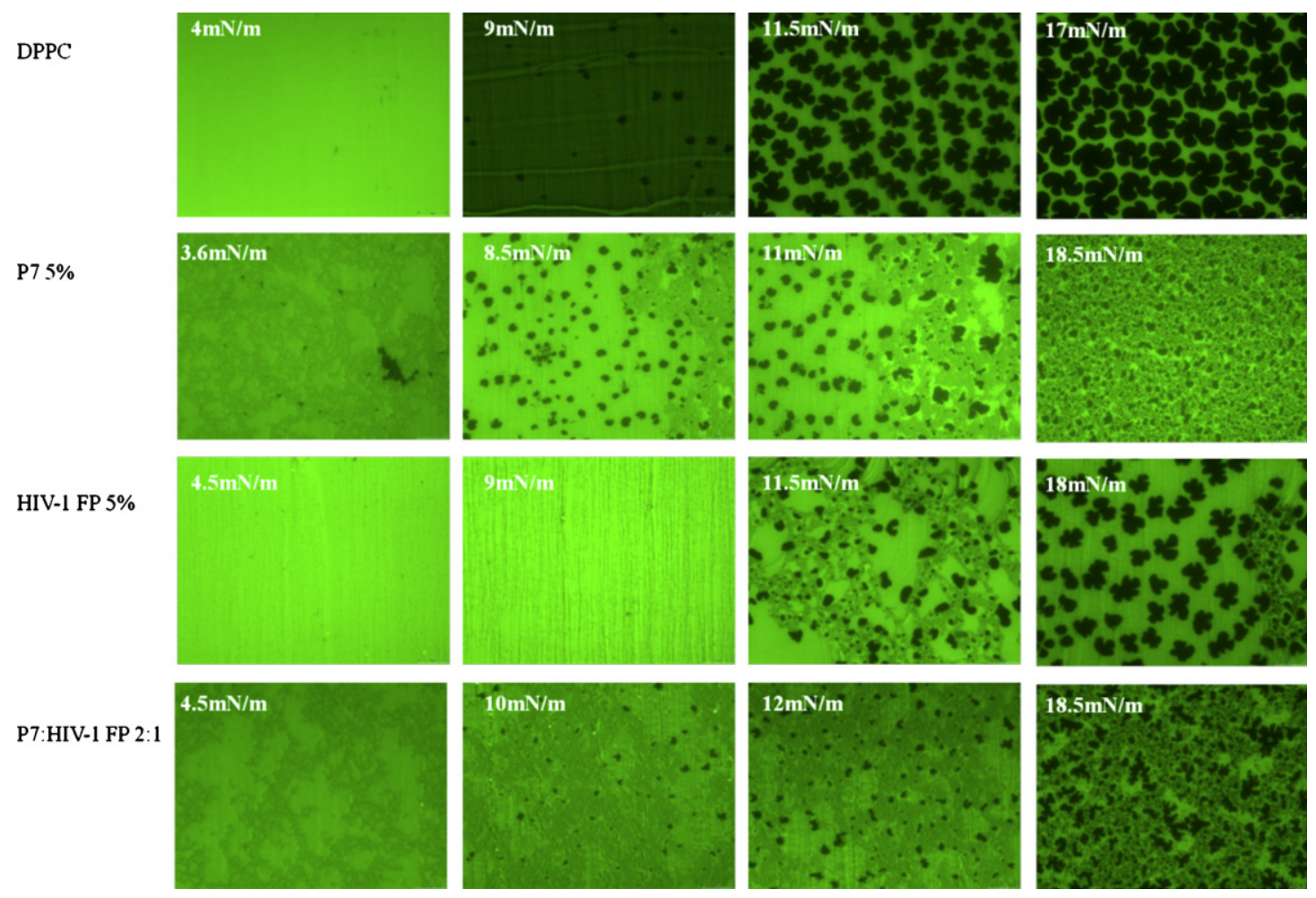

Fig. 6. Epifluorescence images from DPPC monolayers with or without $5 \%$ of peptides P7, HIV-1 FP or the combination 2:1 (mol/mol) of P7:HIV-1 FP. Images were taken at positions matching the indicated surface pressure from the LB films transferred during continuous compression. Images size: $216.56 \mu \mathrm{m} \times 164.96 \mu \mathrm{m}$.

expanded to liquid condensed phase at lower surface pressures than observed in DPPC monolayer, showing more rounded and numerous domains. The images obtained in the presence of any peptide separately show dark percolated regions that could be explained as and increment of the condensed phase of the lipid or even as regions where the peptides segregate. The detection of the presence of the peptide in these regions require of further experiments using fluorescent peptides. The presence of these dark areas
DPPG
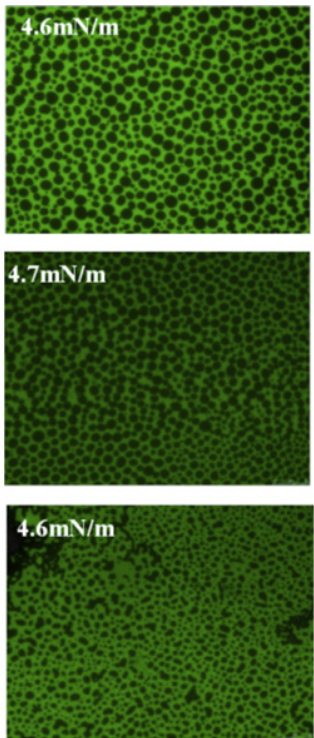

P7:HIV-1 FP 2:

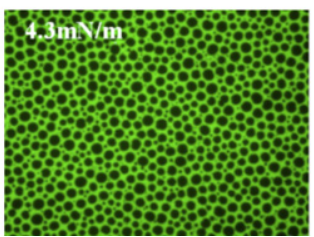

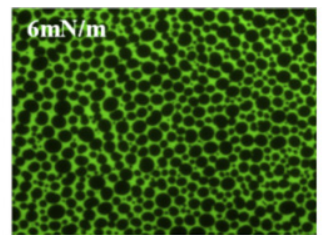
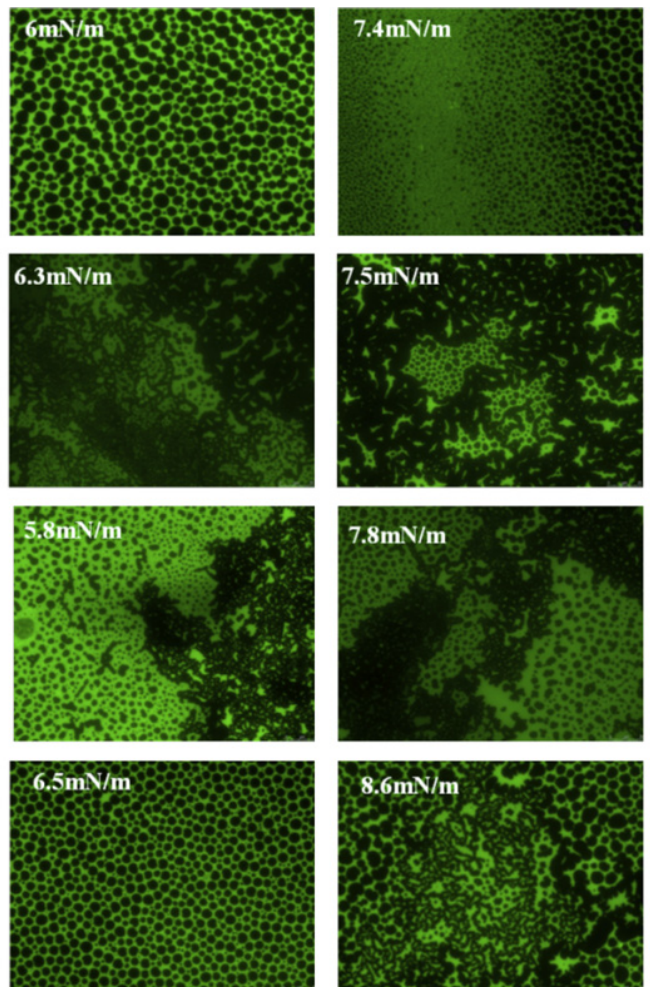
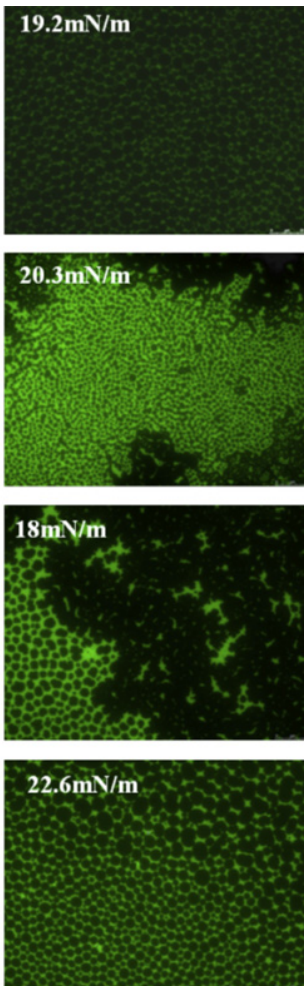

Fig. 7. Epifluorescence images from DPPG monolayers with or without 5\% of P7, HIV-1 FP or a 2:1 (mol/mol) mixture of P7: HIV-1 FP. Images were taking at positions matching the indicated surface pressure from the LB films transferred during continuous compression. Images size: $216.56 \mu \mathrm{m} \times 164.96 \mu \mathrm{m}$. 
coexists with vast regions where the effect of the peptide over the lipid phase transition become more evident appearing as a reduction of the size of the condensed domains, this effect is more patent at higher pressures $(\sim 20 \mathrm{mN} / \mathrm{m})$ where the monolayer of pure DPPG is almost totally condensed. However, if DPPG was prepared in the presence of a 2:1 molar ratio mixture of both peptides, P7 and HIV1 FP respectively, the monolayer seems to be less affected than when each peptide was added separately. The films show domains with regular size and shapes, similar to those present in the pure lipid monolayer, and less dark regions. The combined effect of the peptides in DPPG monolayers could be explained in terms of a peptide/peptide interaction.

\section{Conclusions}

The present investigations try to offer some light on the interaction of four sequences corresponding to E1 protein of GBV-C with the HIV-1 FP in order to get further into the study carried out in a past work. Previously, P10 was discarded as a possible inhibitor of the HIV-1 FP activity and was suggested as a possible fusion peptide of GBV-C (Sánchez-Martín et al., 2011a); these results are now corroborated in this study. We have found that $\mathrm{pI}$ and the charge of P10 are the most close to those of HIV-1 FP as well as the estimation of the content of $\alpha$-helical conformation according to molar ellipticity at $222 \mathrm{~nm}$ is equal for both peptides. Moreover, P10 shows stronger hydrophobic interactions when engaged in an air-water interface than the others, corroborated by Hopp and Woods hydrophobicity profile that is also very similar to HIV-1 FP profile.

P18 was found not to inhibit the interaction with bilayers or the membrane fusion caused by HIV-1 FP. The present results show that P18 is the least hydrophobic of all and it is the only one that, in presence of HIV-1 FP, reduces its percentage of $\alpha$-helix instead of increasing it as the others. Compression isotherms show its high miscibility with the negatively charged phospholipid and how it is capable of reducing the expansion of the mean molecular area caused by HIV-1 FP; thus, we have another evidence of its ability to inhibit the activity of HIV-1 FP in the same way that it was capable of inhibiting the leakage of vesicular contents caused by HIV-1 FP.

P7 and P8 behave very similar. They were capable of inhibiting the leakage of vesicular contents, the membrane fusion and the interaction with bilayers of HIV-1 FP. These results reflect again the similarities between both peptides as the $\mathrm{pI}$ and the net charge, the surface activity curves or the increase of the percentage of $\alpha$ helix when mixed with HIV-1 FP corroborating a conformational change that could be the cause of the inhibition effect. Moreover, the contraction of the mean molecular area observed in the case of HIV-1 FP in presence of P7 indicates that peptide-lipid interactions are attractive and may reflect the formation of peptide-lipid complexes through hydrophobic interactions. The interaction between P7 and HIV-1 FP was also observed by isothermal titration calorimetry were the binding of P7 to HIV-1 FP was found to be endothermic (Sánchez-Martín et al., 2011a). On the contrary, P8 does not seem to change the deviations in the mean molecular area caused by HIV-1 FP nor its interaction with HIV-1 FP in solution was observed by isothermal titration calorimetry.

In conclusion, after several studies to evaluate the possibility of using synthetic peptides corresponding to the envelop protein $\mathrm{E} 1$ of GBV-C in future anti-HIV research, there is one of the peptides studied that excels in all the experiments, P7, that corresponds to the region E1 (19-36); therefore, this peptide could be potentially used in future anti-HIV-1 therapies. P8 and P18 are capable of inhibiting some actions that HIV-1 FP has on membrane models, therefore, we could not discard them and we need to investigate deeply these peptides to understand the reason for this ability and why they do not act in same way in all the experiments assayed.
Being in mind that the action of drugs is at physiological conditions, it is necessary also to investigate the temperature effect on the observed behavior of peptides, mainly for P7 peptide. It is known the conformation of peptides, surface properties and peptide-lipid interaction parameters change with temperature. In case of E1 (145-162) sequence, a new specie appears at a specific molar fraction depending on the temperature (Sánchez-Martín et al., 2009), so its effect cannot be discarded.

\section{Acknowledgements}

This work was supported by project CTQ2009-13969C02/01/BQU from the Secretaría de Estado de Investigación, Ministerio de Ciencia e Innovación, Dirección General de Programas y transferencia de conocimiento, Subdirección General de Proyectos de Investigación (Spain). M.J. Sánchez-Martín is a recipient of an FPI programme pre-doctoral grant. The authors are members of the consolidated research group recognized by the Generalitat de Catalunya "Peptides and Proteins: physicochemical studies” (2009SGR560).

\section{References}

Andrews, M.J.I., Tabor, A.B., 1999. Forming stable helical peptides using natural and artificial amino acids. Tetrahedron 55, 11711-11743.

Bhattarai, N., Stapleton, J.T., 2012. GB virus C: the good boy virus? Trends Microbiol. 20, 124-130.

Bougis, P., Rochat, H., Pieroni, G., Verger, R., 1981. Penetration of phospholipid monolayers by cardiotoxins. Biochemistry 20, 4915-4920.

Brant, D.A., Flory, P.J., 1965. The configuration of random polypeptide chains II. Theory. J. Am. Chem. Soc. 87, 2791-2800.

Chen, Y.H., Yang, J.T., Chau, K.H., 1974. Determination of the helix and beta form of proteins in aqueous solution by circular dichroism. Biochemistry 13,3350-3360.

Deshayes, S., Plenat, T., Aldrian-Herrada, G., Divita, G., Le Grimellec, C., Heitz, F., 2004. Primary amphipathic cell-penetrating peptides: structural requirements and interactions with model membranes. Biochemistry 43, 7698-7700.

Fernández-Vidal, M., Rojo, N., Herrera, E., Gómara, M.J., Haro, I., 2008. Liposome destabilization induced by synthetic lipopeptides corresponding to envelope and non-structural domains of GBV-C/HGV virus, conformational requirements for leakage. Biophys. Chem. 132, 55-63.

Gaines, G.L., 1966. Insoluble Monolayers at Liquid-Gas Interfaces. WileyInterscience, New York, pp. 286.

Galvez, M.J., Cabrerizo, M.A., 1991. A study of the miscibility of bile components in mixed monolayers at the air-liquid interface I. Cholesterol, lecithin, and lithocholic acid. Colloid. Polym. Sci. 269 (1), 77-84.

Greenfield, N.J., 2006. Using circular dichroism spectra to estimate protein secondary structure. Nat. Protoc. 1, 2876-2880.

Linnen, J., Wages Jr., J., Zhang-Keck, Z.Y., Fry, K.E., Krawczynski, K.Z., Alter, H., Koonin, E., Gallagher, M., Alter, M., Hadziyannis, S., Karayiannis, P., Fung, K., Nakatsuji, Y., Shih, J.W., Young, L., Piatak Jr., M., Hoover, C., Fernandez, J., Chen, S., Zou, J.C., Morris, T., Hyams, K.C., Ismay, S., Lifson, J.D., Hess, G., Foung, S.K., Thomas, H., Bradley, D., Margolis, H., Kim, J.P., 1996. Molecular cloning and disease association of hepatitis $\mathrm{G}$ virus: a transfusion-transmissible agent. Science (New York, N.Y) 271, 505-510.

Lobley, A., Whitmore, L., Wallace, B.A., 2002. DICHROWEB: an interactive website for the analysis of protein secondary structure from circular dichroism spectra. Bioinformatics (Oxford England) 18, 211-220.

Nag, K., Boland, C., Rich, N., Keough, K.M., 1991. Epifluorescence microscopic observation of monolayers of dipalmitoylphosphatidylcholine: dependence of domain size on compression rates. Biochim. Biophys. Acta 1068, 157-160.

Nag, K., Perez-Gil, J., Cruz, A., Keough, K.M., 1996. Fluorescently labeled pulmonary surfactant protein C in spread phospholipid monolayers. Biophys. J. 71, 246-250.

Parthasarathy, R., Chaturvedi, S., Go, K., 1995. Design of alpha-helical peptides: their role in protein folding and molecular biology. Prog. Biophys. Mol. Biol. 64, 1-54.

Perczel, A., Hollosi, M., 1996. Turns. In: Fasman, G.D. (Ed.), Circular Dichroism and the Conformational Analysis of Biomolecules. Plenum Press, New York.

Perez-Gil, J., Nag, K., Taneva, S., Keough, K.M., 1992. Pulmonary surfactant protein SP$C$ causes packing rearrangements of dipalmitoylphosphatidylcholine in spread monolayers. Biophys. J. 63, 197-200.

Pérez, J.A. Cantó, J., Reig, F., Pérez, J.., Haro, I. 1998. Conformational behavior of the HAV-VP3 (110-121) peptidic sequence and synthetic analogs in membrane environments studied by CD and computational methods. Biopolymers $45,479-480$.

Rafalski, M., Lear, J.D., DeGrado, W.F., 1990. Phospholipid interactions of synthetic peptides representing the N-terminus of HIV gp41. Biochemistry 29, 7917-7920.

Ramirez-Alvarado, M., Blanco, F.J., Niemann, H., Serrano, L., 1997. Role of beta-turn residues in beta-hairpin formation and stability in designed peptides. J. Mol. Biol. 273, 898-900. 
Sánchez-Martín, M., Amigo, J., Pujol, M., Haro, I., Alsina, M., Busquets, M., 2009. Fluorescence study of the dynamic interaction between E1 (145-162) sequence of hepatitis GB virus C and liposomes. Anal. Bioanal. Chem. 394, 1003-1010.

Sánchez-Martín, M.J., Haro, I., Alsina, M.A., Busquets, M.A., Pujol, M., 2010. A Langmuir monolayer study of the interaction of E1 (145-162) hepatitis G virus peptide with phospholipid membranes. J. Phys. Chem. B 114, 448-450.

Sánchez-Martín, M.J., Hristova, K., Pujol, M., Gómara, M.J., Haro, I., Asunción Alsina, M., Antònia Busquets, M., 2011a. Analysis of HIV-1 fusion peptide inhibition by synthetic peptides from E1 protein of GB virus C. J. Colloid Interf. Sci. 360, 124-130.

Sánchez-Martín, M.J., Urbán, P., Pujol, M., Haro, I., Alsina, M.A., Busquets, M.A., 2011b. Biophysical Investigations of GBV-C E1 peptides as potential inhibitors of HIV-1 fusion peptide. ChemPhysChem 12, 2816-2820.

Simons, J.N., Pilot-Matias, T.J., Leary, T.P., Dawson, G.J., Desai, S.M., Schlauder, G.G., Muerhoff, A.S., Erker, J.C., Buijk, S.L., Chalmers, M.L., et al., 1995. Identification of two flavivirus-like genomes in the GB hepatitis agent. Proc. Natl. Acad. Sci. U.S.A. $92,3401-3410$.
Tillmann, H.L., Manns, M.P. 2001. GB virus-C infection in patients infected with the human immunodeficiency virus. Antiviral Res. 52, 83-90.

Wang, L., Cruz, A., Flach, C.R., Perez-Gil, J., Mendelsohn, R., 2007. Langmuir-Blodgett films formed by continuously varying surface pressure, characterization by IR spectroscopy and epifluorescence microscopy. Langmuir 23, 4950-4960.

Whitmore, L., Wallace, B.A., 2004. DICHROWEB, an online server for protein secondary structure analyses from circular dichroism spectroscopic data. Nucleic Acids Res. 32, 668-670.

Wu, M., Nie, S.-Q., Qiu, Y., Lin, K.-C. Wang, S.-X., Sui, S.-F, 2002. Study of the relationship between structure and function of HIV-1 gp $41 \mathrm{~N}$ terminus fusion peptide. In: Peptides Biology and Chemistry, 104-107.

Xiang, J., George, S.L., Wunschmann, S., Chang, Q., Klinzman, D., Stapleton, J.T., 2004 Inhibition of HIV-1 replication by GB virus C infection through increases in RANTES, MIP-1alpha, MIP-1beta, and SDF-1. Lancet 363, 2040-2050.

Xiang, J., Wunschmann, S., Diekema, D.J., Klinzman, D., Patrick, K.D., George, S.L., Stapleton, J.T., 2001. Effect of coinfection with GB virus C on survival among patients with HIV infection. New Engl. J. Med. 345, 707-710. 